\title{
Optimal Placing of Wind Turbines: Modelling the Uncertainty
}

\author{
Timo Leenman and Frank Phillipson
}

\begin{abstract}
When looking at the optimal place to locate a wind turbine, trade-offs have to be made between local placement and spreading: transmission loss favours local placements and the correlation between the stochastic productions of wind turbines favours spreading. In this paper steps are described to determine the locations of new wind mills that minimize energy loss on the High Voltage power grid. A vindication of the used power grid model is provided, the simulation procedure for stochastic wind power is described and the required mathematical optimization models are described as well as implemented. Results are shown and their relation to real life problems is discussed. The analysis leads to the observation that in reality the entire Dutch coast is popular to locate wind turbines but the only region where this leads to actual reduction of the losses is the North (Groningen and Friesland). Next to this, at the current share of wind energy in the total network load, a spreading strategy to reduce variance of total wind power production does not seem advisable. At higher penetration $(30 \%$ or more) spreading will become important.
\end{abstract}

Index Terms-Optimizing energy generation, wind turbines placement, transportation losses, optimization methods.

\section{INTRODUCTION}

Recent power market developments and the increasing penetration of renewable energy such as wind power, account for growing uncertainties both in demand and in supply of power. This affects not only the issues of power quality and voltage regulation in short term power system operation; it also has consequences for general grid performance measures such as congestion risk and transmission losses. In this paper a new view on the problem is presented and a case study is presented where the relation between wind turbine placement and network losses for the Dutch HV grid is assessed.

In our view, when looking at the optimal place to locate a wind turbine, two trade-offs have to be made:

1) Local or central placement: loss percentage for transportation of a certain amount of electricity is proportional to travelled distance. If all demand and production of power on the grid were deterministic and time-invariant, transportation losses would be minimized by placing all generators as close to demand points as possible. But when demand and production are time-varying or stochastic, sometimes local production is higher than local demand. Local excess power in peripheral parts of the grid has to be transported further than if it were produced at some central point. So local placement of generators is advantageous when local demand is larger than local production, but disadvantageous when local demand is

Manuscript received February 14, 2014; revised June 16, 2014.

Timo Leenman and Frank Phillipson are with the TNO, Delft, the Netherlands (e-mail: frank.phillipson@tno.nl). smaller than local production. In that last case central placement would be better. Therefore loss-minimizing placement of stochastic generators may be regarded as a kind of trade-off between the advantages of local and central placement.

2) Spreading or concentration: the correlation between the stochastic productions of wind turbines reduces when they are placed further apart. So a certain spreading of the wind mills will result in smaller variance of total wind power production, and therefore may reduce the disadvantage of its stochastic nature. However, spreading of the wind mills may have to mean that some are placed in regions with less wind, which in itself is undesirable. Also in this respect a trade-off has to be made.

The main question in this paper is: Given a normal load situation of a HV network, what would be the optimal locations to build a given number of new wind mills, in order to minimize the expected energy transportation losses, considering the presented trade-offs? We present a three step procedure to arrive at the results presented below. The first step is to apply the well-known DC power flow model to the network structure and the load data. The second step is to specify a set of scenarios describing the wind power production behaviour of the wind turbines to be connected to the grid. Each scenario is described by a different wind power output and its proper probability, all based on real wind data. The third step is to optimize expected power losses over all different possibilities of wind turbine placements using a model for stochastic optimization, solved quickly by a novel, very simple, but well justified heuristic.

In Section II an overview is given of the literature related to the topic of this paper. In Section III a short introduction to power grids is provided. A simplified model is derived for the performance of energy transportation through the power grid The principles of electronics are used to arrive at the technical description of power grid behaviour: the so called power flow equations. Some simplifications are applied in order to derive a model in terms of network flow and mathematical programming. Section IV introduces the stochastic optimization model, discussing some relevant aspects and choices made therein. Here we formulate the model in a precise mathematical manner, distinguishing two stages, and adopting terminology from graph theory and mathematical (stochastic) programming. In Section $\mathrm{V}$ the mathematical optimization theory is discussed by which the model formulated in Section IV can be brought into a form that should be solvable by standard optimization software. Because the rigorous optimization quickly becomes too large to perform and because there is quite some structure in the problem that that we can use to provide a solution, a heuristic is presented. The optimization model derived in Sections IV 
and $\mathrm{V}$ introduced some parameters which represent the uncertainty contained in wind power generation. The purpose of Section VI is to perform a simulation in order to estimate these parameters. Section VII discusses the results of the Dutch case and in Section VIII conclusions are drawn from the cases.

\section{LITERATURE}

In order to give an impression of the relation of this research to the topics that are treated elsewhere in literature the contents of some recent publications are discussed. These articles are clustered around the themes network design for loss minimization, wind power modelling and power system expansion.

When searching the scientific literature for minimization of power losses in a network, it becomes clear that there exists a long history of research on this topic, and that it is now vivid as ever. The major part of the research papers is on subjects such as the optimal placement of capacitors on a distribution line, or the optimal configuration of sectionalizing switches in a near-radial distribution system, for example [1]-[3]. Newer is the interest in using Distributed Generation to minimize transportation losses, see for example [4]-[8]. While the field of renewable energy is constantly growing, and wind power is one of its largest contributors a lot of research is done into wind power modelling under two main themes: firstly, steady state analysis, which is mainly used in long term planning, power grid design and generation expansion studies; and secondly, dynamic analysis (or, more commonly: forecasting). This is used for short term prediction of future wind power generation, in order to optimize intraday market biddings and short term power system control. Especially because of the reactive power behaviour of wind turbines, voltage stability in the system may depend crucially on their correct operation. Examples of earlier work are [9]-[13].

A more holistic view, namely the inclusion of wind turbines in an existing grid is found in [14], who addresses the question of "distribution system planning in the face of a worldwide growth of DG penetration". The aim of their article is to present a "proper tool, able to find the siting and sizing of DG units which minimize generalized cost" as a help for the distribution system planner. They designed a three-step algorithm to find the optimal locations and sizes of several wind turbines, to be built within the area of some Medium Voltage (MV) distribution grid. The first step consists of an implementation of the method of [12] mentioned above; the second step is a genetic algorithm which steadily improves the intermediate solution. The objective function (generalized cost) contains several terms, such as location dependent building costs, but also the cost of power losses. The third step is a method of determining the robustness of a solution under uncertain future conditions. Lastly, they apply the method to a particular Italian MV grid.

Ref. [15] discusses applications from design in logistic networks and power systems. The subject of chapter 4 is Transmission and Generation Expansion Planning (TGEP) for wind farms in power systems. He shows that for wind energy, the expansion planning of generation capacity and of transmission capacity should be considered simultaneously.
He shows that however very little work has been done on the simultaneous TGEP problem, mostly generation and transmission expansion are regarded independently, or else a deterministic TGEP model is used. But wind power requires a stochastic model.

A total different approach is found in [16]. This paper presents an approach to calculate the optimal location of renewable energy generation. Potential areas for the expansion of the considered generation technologies (wind onshore and offshore, photovoltaic and concentrated solar power) are calculated in a land use analysis. The corresponding renewable energy supply is determined via a meteorological analysis. Eventually optimal RES investment decisions are determined. Wind energy should mainly be expanded on coastlines but higher capacities need also efficient interior locations.

Although this research is related to [15] there are some differences in the approach. We go into much more detail of the power grid and use a different model for it. Next to that, as our model is very complex and simplification does not help enough, we present a simple heuristic to generate solutions. Our approach, looking at transportation losses and taking into approach the correlation in wind power production lead to conflicting conclusions.

\section{POWER GRID MODELLING}

In this section an introduction into power grids is provided. Then the power flow equations are formulated, energy loss is described, and by several steps a simplified model for power grid behaviour is derived.

\section{A. Power Grids}

A power grid is a set of electrical components called buses, which are connected with each other by transmission lines, called branches. Some of the buses represent electricity consumers, which have a demand for electrical power. These buses are called load buses. Some other buses represent power plants, or other sources of electrical power. These buses are called generation buses. In order to keep the voltages through the entire grid at their demanded level, the system is operated in such a way that supply equals demand at every point in time. As demand varies in time depending on the wishes of the consumers, power generation has to be adjusted accordingly.

Due to resistance of transmission lines, electrical energy is lost during transportation from generation to load buses. These losses are reduced by applying higher voltages, but electricity has to be delivered to consumers at only 230V. As a result, most power grids are designed in the following way:

1) High Voltage grid, or transmission grid. It is kept at voltages of $110 \mathrm{kV}$ to $450 \mathrm{kV}$.

2) Medium Voltage grid, being the link between transmission and distribution. It is kept at voltages between $1 \mathrm{kV}$ and $110 \mathrm{kV}$.

3) Low Voltage grid, or distribution grid. Voltage is below $1 \mathrm{kV}$.

\section{B. Power Flow Equations and Power Loss}

The usual physical representation of a transmission line is:

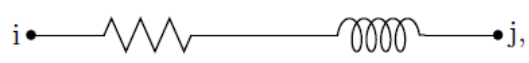


that is, an ideal line $(i j)$ containing a resistance $r_{i j}$, and a reactance $x_{i j}$. The impedance of this line then, is $Z_{i j}=r_{i j}+i x_{i j}{ }^{1}$. This means that the relation between current and voltage in the line can be described by Ohm's law $I_{i j}=\left(V_{i}-V_{j}\right) / Z_{i j}$. Assuming a complex voltage of $V_{i}=v_{i} e^{i \theta_{i}}$ at bus $i$, and of $V_{j}=v_{j} e^{i \theta_{j}}$ at bus $j$, yields exercised powers of

$$
\begin{gathered}
S_{i j}=V_{i} I^{*}=V_{i} \frac{V_{i}^{*}-V_{j}^{*}}{Z^{*}}=\frac{v_{i}^{2}-v_{i} v_{j} e^{i\left(\theta_{i}-\theta_{j}\right)}}{r-i x}, \\
S_{j i}=\frac{v_{j}^{2}-v_{i} v_{j} e^{i\left(\theta_{j}-\theta_{i}\right)}}{r-i x} .
\end{gathered}
$$

Note that for ease of notation from here on $r$ and $x$ will be written instead of $r_{i j}$ and $x_{i j}$, because the context makes clear which resistances and reactances are meant.

Adding the complex powers exercised by voltages $V_{i}$ and $V_{j}$ at both ends of the line yields a total complex power conversion of

$$
S_{t o t}=S_{i j}+S_{j i}=\frac{v_{i}^{2}+v_{j}^{2}-v_{i} v_{j}\left(e^{i\left(\theta_{i}-\theta_{j}\right)}-e^{i\left(\theta_{j}-\theta_{i}\right)}\right)}{r+i x} .
$$

The magnitude $\left|S_{i j}\right|$ of a complex power is called the apparent power. Because of Joule's law $Q=I^{2} \Omega t$, it is the current which determines the capacity of a transmission line (before it is damaged by heat production). Moreover for fixed voltage magnitude, the apparent power $\left|S_{i j}\right|=\left|V_{i} I^{*}\right|=\left|V_{i}\right||I|$ is proportional to the current on a line. Therefore in a power grid line capacities can be expressed in maximum apparent power.

But the apparent power says nothing about the power conversion in the line. That can be seen by taking the real parts of the above expressions, using $\cos _{i j}(\theta)=\cos \left(\theta_{i}-\theta_{j}\right)$ and $\sin _{i j}(\theta)=\sin \left(\theta_{i}-\theta_{j}\right)$ representing the real powers exercised by voltages $V_{i}$ and $V_{j}$ :

$$
\begin{aligned}
& p_{i j}=\frac{1}{r^{2}+x^{2}}\left[r v_{i}^{2}-r v_{i} v_{j} \cos _{i j}(\theta)+x v_{i} v_{j} \sin _{i j}(\theta)\right], \\
& p_{j i}=\frac{1}{r^{2}+x^{2}}\left[r v_{j}^{2}-r v_{i} v_{j} \cos _{i j}(\theta)-x v_{i} v_{j} \sin _{i j}(\theta)\right],
\end{aligned}
$$

And adding them yields a total real power conversion of

$$
p_{l o s s}=p_{i j}+p_{j i}=\frac{1}{r^{2}+x^{2}}\left[r\left(v_{i}^{2}+v_{j}^{2}\right)-2 r v_{i} v_{j} \cos _{i j}(\theta)\right]
$$

This formula represents energy loss per second, depending on complex voltages on both ends of the line.

If we take the imaginary parts of the complex powers, we get the so called reactive powers

$$
q_{i j}=\frac{1}{r^{2}+x^{2}}\left[x v_{i}^{2}-r v_{i} v_{j} \sin _{i j}(\theta)-x v_{i} v_{j} \cos _{i j}(\theta)\right],
$$

\footnotetext{
${ }^{1}$ Capitals are used to represent complex quantities.
}

$$
q_{j i}=\frac{1}{r^{2}+x^{2}}\left[x v_{j}^{2}+r v_{i} v_{j} \sin _{i j}(\theta)-x v_{i} v_{j} \cos _{i j}(\theta)\right] .
$$

And these four equations describe the total (steady state) power grid behaviour, because Kirchhoff's Current Laws demand power flow conservation at every bus $i$ for real power (that is, $\sum_{j} p_{i j}-\sum_{j} p_{j i}=0$ ), and also for reactive power (that is, $\sum_{j} q_{i j}-\sum_{j} q_{j i}=0$ ). By specifying certain boundary values at the buses (for example, $p_{i j}$ and $v_{i}$ at a generation bus $\underline{i}$ ), and one reference node with phase $\theta=0$, one can then solve for all voltage magnitudes and phases in the system. This is called AC power flow calculation.

\section{DC Load Flow Model}

For technical purposes it is often required to be able to calculate the behaviour of bus voltage magnitudes and angles. It is important to maintain voltage stability, even when power demand and generation is subject to change. Unexpected reactive power behaviour may result in local voltage drops (which is undesirable for customers), or voltage rises (which may damage the electrical components of the system). Therefore the full solution of the above non-linear equations is critical for the technical operation of the system. However for general design questions and performance estimations, it is expedient to simplify a little further. Ref. [17] justifies the following assumptions for the context of this paper:

1) The bus voltage magnitudes $v_{i}$ and $v_{j}$ are almost equal, so that we can say $v: \approx v_{i} \approx v_{j}$. That is, the effect of voltage drop on power system behaviour is neglected. Certainly for high voltage systems this is justified.

2) The difference in voltage angles $\left(\theta_{i}-\theta_{j}\right)$ is small. In this case one can make the first order approximations $\sin _{i j}(\theta) \approx\left(\theta_{i}-\theta_{j}\right)$ and $\cos _{i j}(\theta) \approx 1$.

3) An unnecessary assumption for our modeling purposes, but nevertheless true in practice for HV transmission lines, is $r \ll x$ (resistance is much smaller than reactance).

Making these approximations, and putting $v=1$ (which is called expressing the voltage in per-unit) yields for our real power equations

$$
\begin{gathered}
p_{i j}=\frac{1}{r^{2}+x^{2}}\left[r v_{i}^{2}-r v_{i} v_{j} \cos _{i j}(\theta)+x v_{i} v_{j} \sin _{i j}(\theta)\right] \\
\approx \frac{1}{r^{2}+x^{2}}\left[r v^{2}-r v v+x v^{2}\left(\theta_{i}-\theta_{j}\right)\right] \\
=\frac{x}{r^{2}+x^{2}}\left(\theta_{i}-\theta_{j}\right) \approx \frac{1}{x}\left(\theta_{i}-\theta_{j}\right), \\
p_{j i} \approx \frac{x}{r^{2}+x^{2}}\left(\theta_{j}-\theta_{i}\right) \approx \frac{1}{x}\left(\theta_{j}-\theta_{i}\right) .
\end{gathered}
$$

One can see that due to $v_{i}=v_{j}$, the first and second term in square brackets cancel out. The justification of this approximation for HV power grid is even more enhanced by the third assumption that $x$ tends to be (much) larger than $r$, making the third term dominant already.

Now compare a purely resistive DC electrical circuit where the only active elements are current sources connected 
to ground. In this case the current flow equations are $I_{i j}=\left(V_{i}-V_{j}\right) / R_{i j}$ for all lines $(i j) .^{2}$

Note the similarity between the simplified power equations of the AC power grid, and the current equations of the DC electrical circuit. Their structure is exactly the same, only quantities differ. Thus it can be seen that after these simplifications a remarkable analogy exists between a large $\mathrm{AC}$ power system, and a DC electrical circuit equivalent. The role of currents in the DC load flow is taken over by powers in the $\mathrm{AC}$ network; the role of DC voltage drops over resistors correspond to differences between bus voltage angles, and DC resistance values correspond to reactance $x$ of a transmission line.

It is according to this analogy that we like to think about the power grid as if there are power sources, power sinks, a power flow through the network, (even 'power conservation laws'), en passant producing line losses.

\section{Reactive Power and Line Loss in the DC Model}

The same approximations 1. and 2. from the previous section, applied to the reactive power equations, yield:

$$
\begin{gathered}
q_{i j}=\frac{1}{r^{2}+x^{2}}\left[x v_{i}^{2}-x v_{i} v_{j} \cos _{i j}(\theta)-r v_{i} v_{j} \sin _{i j}(\theta)\right] \\
\approx \frac{1}{r^{2}+x^{2}}\left[x v^{2}-x v v+x v^{2}\left(\theta_{i}-\theta_{j}\right)\right]=\frac{-r}{r^{2}+x^{2}}\left(\theta_{i}-\theta_{j}\right), \\
q_{j i} \approx \frac{-r}{r^{2}+x^{2}}\left(\theta_{j}-\theta_{i}\right)=\frac{r}{x} p_{i j},
\end{gathered}
$$

that is, if also $r<<x$, reactive power 'flows' vanish, or at least are neglectable in comparison to real power flows.

Similarly, the first order approximation for real power loss yields $p_{\text {loss }}=p_{i j}+p_{j i}=0$. The DC model assumptions formulated at the begining of the previous paragraph have as a direct consequence that zero power loss is assumed. This can easily be verified by the consideration that for all $i$ and $j$, $v_{i}=v_{j}$, which means that there is zero voltage drop in the system, and consequently there can be no net power conversion. But taking the second order approximation $\cos (\theta) \approx 1-\frac{\theta^{2}}{2}$ produces

$$
\begin{aligned}
& p_{\text {loss }}=\frac{1}{r^{2}+x^{2}}\left[r\left(v_{i}^{2}+v_{j}^{2}\right)-2 r v_{i} v_{j} \cos _{i j}(\theta)\right] \\
& \approx \frac{1}{x^{2}+r^{2}}\left[2 r-2 r\left(1-\frac{\left(\theta_{i}-\theta_{j}\right)^{2}}{2}\right)\right] \approx r p_{i j}^{2}
\end{aligned}
$$

These powers do not actually disappear from the power flows in the model (which are, like the current in an electrical circuit, preserved), and therefore do not influence the power flow solution.

\section{E. Network Flow Model}

Above it was established by simplifying the exact AC power flow equations, that large scale $\mathrm{AC}$ power flow behaves approximately like a simple small scale DC electrical circuit. In former times this similarity was used by

\footnotetext{
${ }^{2}$ Here capitals represent normal real DC quantities.
}

the old "DC network analyzer", in which each network branch was represented by a resistance proportional to its series reactance and each DC current was proportional to a real power flow. The DC model derived its name from this analogue computing table.

These days of course computer algorithms can be exploited. By basic physics of electricity, Kirchhoff's Current Laws (KCL) and Kirchhoff's Voltage Laws (KVL) yield the equations by which the solution for the nodal voltages and currents over the lines can be computed. KCL demand that the total current into a node must equal the total current out of that node. KVL demand that the directed sum of the voltage drops over every closed loop in the network must equal zero. By simple linear algebra techniques the resulting system of linear equations can be solved. In power flow analysis, the matrix of coefficients of the linear equations is called the admittance matrix. The solution procedure comes down to inverting this matrix.

In the context of this paper (stochastic and constrained optimization) another approach is more useful and also more intuitive. Ref. [18] remarks that the currents found by solving both Kirchhoff's Current and Voltage Laws, happen to be the same currents that minimize the total heat dissipation described by $\sum\left(I_{i j}\right)^{2} r_{i j}$, while satisfying only Kirchoff's Current Laws.

Because in the terminology of network flow optimization these current laws are just the flow balances at a node and because the objective function that describes total heat dissipation is clearly separable and convex (quadratic), we know from theory on network flows like [19] that the power flow problem can be solved efficiently by network flow algorithms. The advantage of this method over the traditional former one is that network algorithms allow for straightforward sensitivity analysis, can cope with additional contraints that may come in, and offer a known framework for a stochastic extension.

Concretely, this means that to find the solution for the currents through a DC network, and therefore an approximate solution for the power flow in an AC power system, the following mathematical program should be solved:

$$
\begin{gathered}
\text { Minimize } \\
\text { Subject to } \quad \sum_{(i j) \in A} p_{i j}^{2} x_{i j} p_{i j}-\sum_{j:(j i) \in A} p_{j i}=b_{i} \quad i \in N, \\
p_{i j} \geq 0
\end{gathered}
$$

where $p_{i j}$ is the power flow through line $(i j), x_{i j}$ is the reactance of the line, and $b_{i}$ is the power supply/demand (the balance) of each node $i$.

From this it can be seen that the problem of computing the power flow solution of a power grid, is equivalent to solving a so called minimum cost flow problem with quadratic arc costs. Recall (e.g. from [19]) that the flow balance constraints may be written as $B \vec{p}=b$, where $B$ is the so-called node-arc incidence matrix of the network, $\vec{p}$ is the vector of power flows through the arcs, and $b$ is the vector of supplies at the nodes. Because of notational conventions, in the next chapters we will use $y_{i j}$ instead of $p_{i j}$ as symbol for the power flows. 


\section{F. Additional Remarks}

For the application in this paper equal voltages are assumed throughout the grid. But when this is not the case, the above network flow program can easily be adjusted to account for the voltage differences. Define all arc cost coefficients $c_{i j}=x_{i j} / v_{i j}$ where $v_{i j}$ is the voltage at which the line is kept. The intuitive interpretation of this is, that the high voltage lines are 'cheaper' to travel over, and therefore are more likely to attract flow than lower voltage lines. This is nice since higher voltage lines typically cause less power loss, and have higher capacities.

The application of network algorithms to solve the DC power flow problem seems to be little advocated in literature. Yet modern day algorithms such as network simplex and $\varepsilon$ -relaxation can solve quadratic cost flows extremely efficiently. It has been suggested that these methods are faster than the admittance matrix inversion of classical DC algorithms (e.g., [19], chapter 1). Moreover, strongly polynomial algorithms have been developed for separable quadratic cost generalized flows. In such network problems, a so-called arc multiplier is added to all arcs. The outgoing flow of the arc is then defined as the incoming flow times the arc multiplier. The qrelaxg algorithm, together with mathematical proof of its polynomiality, is presented in [20].

This opens up the possibility to a DC algorithm where power losses are incorporated by a linear (first order) approximation of the losses over a branch. Compare [21] where a zero'th order approximation is suggested for the losses by subtracting fixed loss estimates from the node balances. There it is proposed (Section VII-B) that incorporation of the losses could be achieved by estimating all powerflows $p_{i j}$ beforehand by $\hat{p}_{i j}$. Then $r_{i j} \hat{p}_{i j}^{2}$ estimates the losses over this line. These losses can be subtracted from the node balances of the adjacent nodes before computing the power flows. According to [21], the losses "usually converge" when this process is iterated (using the resulting power flow as the new estimates $\hat{p}_{i j}$ ).

The quadratic cost generalized flow however, immediately yields losses that are proportional to the power flow over the branch. To determine the arc multipliers one should still estimate the power flows beforehand. Then, based on the estimate $\hat{p}_{i j}$, put the arc multiplier $\gamma_{i j}=r_{i j} \hat{p}_{i j}$ such that the linear loss $\gamma_{i j} p_{i j}=r_{i j} \hat{p}_{i j} p_{i j}$ approximates the actual loss $r_{i j} p_{i j}^{2}$. The network programming formulation looks like:

$$
\begin{gathered}
\text { Minimize } \\
\text { Subject to } \quad \sum_{(i j) \in A} p_{i j}^{2} x_{i j} p_{i j}-\sum_{j:(j i j) \in A} \gamma_{j i} p_{j i}=b_{i} \quad i \in N, \\
p_{i j} \geq 0 \quad(i j) \in A
\end{gathered}
$$

and take arc multipliers $\gamma_{i j}=r_{i j} \hat{p}_{i j}$. Iteration by using the resulting $p_{i j}$ as the new flow estimates $\hat{p}_{i j}$, can be expected to converge both faster and more likely than the method of [21] because linear approximation of the quadratic loss function is significantly better than approximation by a constant function.

The model can be improved yet one step further. As will be shown in chapter 5 , every quadratic network flow can be approximated to any desired degree of accuracy by a linear network flow in which parallel arcs are added. The flows over these parallel arcs sum up to the flow over the original quadratic cost arc. When decreasing arc multipliers are associated with these parallel arcs, a piecewise linear approximation of the quadratic loss can be realized. Thus it can be seen that the network flow approximation to the DC power flow problem is flexible enough to realize the incorporation of quadratic losses into the DC power flow equations. We have seen this remark nowhere else in literature. It would be interesting to assess if this improvement makes the DC power flow model more suitable for MV grids, or very large scale HV systems, where power losses are more substantial.

\section{Mathematical Model FoR the Placement PROBLEM}

\section{A. General Outline}

We expect HV and MV power grids to satisfy our DC load flow assumptions, and because the supply and demand of power have to be equal at every point in time, network behavior of the HV/MV network can be modeled by solving a minimum cost flow. The node balances required by such a model can be obtained from the load data of the power network in the Netherlands. These data can be taken from a moment of peak load or of average load. The connection of a wind mill to the grid corresponds to the increase of the supply at that particular node. In practice however, wind power production is highly uncertain. Although power demand by consumers also involves uncertainty, it is far better to predict than wind power production, and for that reason the assumption of deterministic demand seems justified. This assumption is also made by [14].

In Fig. 1 a rough sketch is provided, which may help to visualize the model described. In the original situation, power supply by the power plants (which are mainly connected to the upper parts of the grid) equals total demand by consumers (which are located mainly in the lower parts of the grid). The wind mills to be placed provide an extra (stochastic) supply.

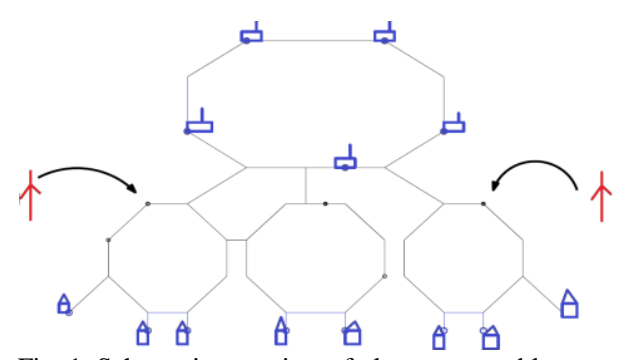

Fig. 1. Schematic overview of placement problem.

Of course, wind speed is geographically correlated: a strong wind in one place makes a strong wind in another place, $100 \mathrm{~km}$ away, more likely. In order to model wind speed distribution over the Netherlands, we shall use wind speed measurements throughout the country to partition the Netherlands into several zones. Within these zones we will assume wind speed to be $100 \%$ correlated, and we shall use the measurement data to estimate the correlations between the zones. 
A few things can be observed: first, because power supply and demand always have to be equal, a choice has to be made what to do with the excess power in the system, resulting from the wind turbines we place. A few possibilities are: sell excess power abroad; increase demand of some demand node(s); decrease generation of some supply nodes. In this last case a choice has to be made which power plants to adjust Because in practice market balancing happens non-locally, we propose to adjust for every MW of wind power, certain other power plants distributed over the country.

Second: in general wind mills are placed 'lower' on the grid than normal power plants. So as long as wind power production does not exceed local demand, wind turbines will have a positive effect on the transportation losses.

Third: there is no intrinsic mechanism in the model that promotes placing the wind turbines on a spot with more wind than elsewhere (except when it can fulfil local demand). This might seem counterintuitive, but it is the consequence of our model. Models that minimize operating costs, do promote windy spots, because wind mills have high building costs but low marginal costs. Therefore windy spots will make more profitable investments.

Fourth: The model does promote spreading the wind mills over different zones, but only if peak wind power production is higher than local demand. In that case the peak spreading results in less power transport, and therefore less energy losses.

In the following two sections first the model is described for computing the power flow and line losses, once all power productions are given. This may be called the second stage of the problem, because wind mills have to be placed first. Next the model is described for the placement of the wind mills, and for computing the produced wind power and node balance corrections. This may be called the first stage of the problem. Note that the second stage will be described prior to the first stage.

\section{B. Power Flow Problem}

Define an (undirected) graph $G=(N, A)$, taking a node $n \in N$ for every bus in the power grid, and an arc $a \in A$ for every transmission line connecting two buses. Define a cost function $c: A \rightarrow R^{\geq 0}$, which associates with every arc $a_{i j} \in A$ a cost $c_{i j}$, and take a cost $c_{i j}$ equal to the reactance $X_{i j}$ of its corresponding transmission line. Define a balance function $b: N \rightarrow R$, associating with every node $i$ its supply of power $b_{i}$ : a negative number for load buses, a positive number for generation buses. Define a nonnegative power flow $y: A \rightarrow N$ on the arcs of $G$, satisfying the balance equation $\sum_{j:(i j) \in A} y_{i j}-\sum_{j:(j i) \in A} y_{j i}=b_{i}$ for each node $i$, and $y_{i j} \geq 0$ for all $(i j) \in A$. Assuming a power balance $\sum_{i \in N} b_{i}=0$, the so called power flow solution is found by minimizing $\sum_{(i j) \in A} c_{i j} y_{i j}^{2}$ over all feasible power flows. ${ }^{3}$ Once the power flows have been found, $\sum_{(i j) \in A} y_{i j}^{2} r_{i j}$ is the expression for

\footnotetext{
${ }^{3}$ Note that it is necessary that the balances sum up to zero, or, total power production equals total power consumption. This is because the DC power flow model assumes that no power losses occur. Power losses are neglected while computing the power flow solution. Afterwards, when the power flow solution has been computed, an estimate is made of the losses that have occurred.
}

the total power loss.

\section{Wind Turbine Placement Problem}

Partition the node set $N$ into $k$ zones such that $N=N_{1} \cup N_{2} \cup \ldots \cup N_{k}$. Introduce the random vector $\tilde{M} \in R_{\geq 0}^{k}$, representing the uncertain wind speeds in the zones at some arbitrary time instance; its $k$-th element $\tilde{M}_{k}$ being the wind speed in zone $k$. Transform $\tilde{M} \in R_{\geq 0}^{k}$ into $M \in R_{\geq 0}^{n}$ by firstly, computing for each zone $i=1, \ldots k$ what wind power is produced by one wind turbine from the wind speed in zone $i$, and secondly, copying this number for each node in that zone. That is, if two nodes $i, j$ are in the same zone, then $M_{i}=M_{j}$. We call $M_{i}$ the potential wind speed at node $i$. Introduce decision variables $x_{i}: i \in N$, representing the choice of placement for the wind turbine in zone $i$. Therefore for all regular $i: x_{i} \in\{0,1, \ldots\}$ and $\sum_{i} x_{i}=l$ should be satisfied, where $l$ is the number of wind mills to be placed. ${ }^{4}$ Now $\sum_{i \in N} M_{i} x_{i}=(\vec{M} \cdot \vec{x})$ represents the total wind power produced, so if $t \in R^{n}$ represents the fractions of production adjustments to compensate for the wind power (with $\left.\sum_{i \in N} t_{i}=1\right)$, then $b_{i}+M_{i} x_{i}-(\vec{M} \cdot \vec{x}) t_{i}$ is the new balance for any node $i \in N$. Note that

$$
\begin{aligned}
& \sum_{i \in N}\left(b_{i}+M_{i} x_{i}-(\vec{M} \cdot \vec{x}) t_{i}\right)= \\
& \left(\sum_{i \in N} b_{i}\right)+\left(\sum_{i \in N} M_{i} x_{i}\right)-\sum_{i \in N}(\vec{M} \cdot \vec{x}) t_{i}= \\
& 0+\vec{M} \cdot \vec{x}+(\vec{M} \cdot \vec{x}) \sum_{i \in N} t_{i}=0
\end{aligned}
$$

So total power balance is indeed always preserved, no matter what the value of $\vec{M}$ may happen to be. Then given a placement vector $\vec{x}$ and a realisation $\vec{m}$ of $\vec{M}$, the vector of power flows $\vec{y}$ can be computed as follows:

$$
\vec{y}=\operatorname{argmin} \sum_{(i j) \in A} c_{i j} y_{i j}^{2}
$$

Subject to

$$
\begin{gathered}
y_{i j} \geq 0 \quad(i j) \in A \\
\sum_{j:(i j) \in A} y_{i j}-\sum_{j:(j i) \in A} y_{i j}=b_{i}+m_{i} x_{i}-(\vec{m} \cdot \vec{x}) t_{i} \quad i \in N .
\end{gathered}
$$

It is clear that the power flows $y_{i j}$ depend on the placement decisions $x_{i}$ and on the random vector $M$, which determine the balances of the minimum cost flow. Hence, different choices of $x_{i}$ will mount to different values of $y_{i j}$, and therefore different transportation losses. Moreover, the expected value of $y_{i j}(\vec{M}, \vec{x})$ over $\vec{M}$ is well defined. The aim then to minimize expected transportation losses by optimally placing the wind mills may be written in the following way:

\footnotetext{
${ }^{4}$ Do not confuse this decision variable $x_{i}$ with the symbol $x_{i j}$ or $x$, used in the previous chapter to denote line reactance. Notational conventions in power engineering demand the use of $x_{i j}$ for reactance, and in mathematical programming it is customary to denote first stage decision variables by $x_{i}$.
} 
Minimize

$$
\mathrm{E}_{M}\left[\sum_{(i j) \in A} y_{i j}^{2} r_{i j}\right]
$$

Subject to

$$
x_{i} \in\{0,1, \ldots\} \quad i \in N
$$

\section{The Complete Model}

Combining the two previous sections, a complete formulation is provided by

Minimize $\quad \mathrm{E}_{M}\left[\sum_{(i j) \in A} y_{i j}^{2} r_{i j}\right]$

Subject to

$$
x_{i} \in\{0,1, \ldots\}
$$

and where

$$
\vec{y}=\arg \min \sum_{(i j) \in A} c_{i j} y_{i j}^{2}
$$

Subject to $\quad y_{i j} \geq 0 \quad(i j) \in A$

$$
(B y)_{i}=b_{i}+m_{i} x_{i}-(\vec{m} \cdot \vec{x}) t_{i} \quad i \in N
$$

In the last equation (which represents the flow balance constraints), $B$ is the node-arc incidence matrix for $G$. The right-hand side $b_{i}+m_{i} x_{i}-(\vec{m} \cdot \vec{x}) t_{i}$ are the balances, adjusted for the placement of the wind turbines.

\section{REFORMULATION INTO DETERMINISTIC OPTIMIZATION PROBLEM}

In Section IV Part D a complete mathematical formulation of the research question was given. In this section we try to reformulate the problem into a standard solvable minimization problem. First, the stochastic program is rewritten into a large deterministic one by introducing scenario parameters. These parameters will be obtained by simulation. Next, a linearisation of the network flow that arises in the second stage is described. Then it is shown how duality theory of linear network flows may be used to combine the two stages into one large optimization problem. Finally, we present the solution strategies for this problem.

\section{A. Stochastic Programming}

Models like the one above are called two stage stochastic optimization problems:

1) First stage: the decisions $\vec{x}$ have to be made while the future behaviour of $M$ is still uncertain; then a realization of $M$ is observed;

2) Second stage the laws of power electronics determine the decision variables $\vec{y}$, which results in a value of the objective function, that is, the transportation losses.

The first difficulty in carrying out the minimization above has to do with the form of the objective function. Deterministic optimization algorithms cannot trivially handle an objective function like

$$
\mathrm{E}_{M}\left[\sum_{(i j) \in A}\left(y_{i j}(\vec{M}, \vec{x})\right)^{2} r_{i j}\right]
$$

which contains an expectation. (The notation $y_{i j}(\vec{M}, \vec{x})$ makes the dependence clear of the $y_{i j}$ on $\vec{M}$ and $\vec{x}$.) The random variable $\vec{M}$ has a continuous (multi-dimensional) distribution, and it is difficult to see how a (multidimensional) integral in the objective can be dealt with. Next to this, another possibility is solving this as a Stochastic Recourse Model. However, then we need both a linearization of the objective function and a relaxation of the integer decision variables.

Every continuous distribution can be approximated by a discrete one, and moreover, one which assumes finitely many values. Therefore also our wind speed distribution may be assumed discrete. And when it is assumed that $\vec{M}$ takes only finitely many different values, the expectation can be computed as a finite sum. To this end, define a set $S$ containing finitely many so-called scenarios $S \in S$. Next assume that $\vec{M}$ will take the value $\vec{m}^{s}$ with probability $p^{s}$, where $\sum_{s \in S} p^{s}=1$. Then the expectation of a function $f(\vec{y})$ of $\vec{y}$ can be written $\sum_{s \in S} p^{s} \times f\left(\vec{y}^{s}\right)$.

In this way the stochastic program of the previous section can be written as an equivalent (large-scale) deterministic extension by summing over the scenario space:

Minimize

$$
\sum_{s \in S} p^{s} \times\left(\sum_{(i j) \in A}\left(y_{i j}^{s}\right)^{2} r_{i j}\right)
$$

Subject to

$$
\begin{gathered}
x_{i} \in\{0,1, \ldots\} \quad i \in N \\
\sum_{i \in N} x_{i}=l,
\end{gathered}
$$

and where

$$
\vec{y}^{s}=\operatorname{argmin} \sum_{(i j) \in A} c_{i j}\left(y_{i j}^{s}\right)^{2}
$$

Subject to

$$
\begin{gathered}
y_{i j}^{s} \geq 0 \quad(i j) \in A, s \in S \\
\left(B \vec{y}^{s}\right)_{i}=b_{i}+m_{i}^{s} x_{i}-\left(\vec{m}^{s} \cdot \vec{x}\right) t_{i} \quad i \in N, s \in S .
\end{gathered}
$$

\section{B. Linearization}

Still this formulation does not fit a standard mathematical programming model. The first stage decisions $\vec{x}$ aim to minimize the expectation of some function of the second stage decisions $\vec{y}^{s}$, whereas these themselves must be chosen in such a way that they minimize some other function. At first sight it is not clear how to formulate the entire problem as a single optimization model. The constraint that the $\vec{y}^{s}$ are the

\footnotetext{
${ }^{5}$ The scenario set $S$ is a kind of finite discretization of the sample space $\Omega$ of $M$ : in fact, the expectation in the objective function could have been written $\int_{\omega \in \Omega_{M}} \cdot\left(\sum_{(i j) \in A}\left(y_{i j}^{\omega}\right)^{2} r_{i j}\right) d \omega$, whereas the approximation by introducing the scenario set $S$ looks like $\sum_{s \in S} p^{s} \cdot\left(\sum_{(i j) \in A}\left(y_{i j}^{s}\right)^{2} r_{i j}\right)$.
} 
arguments which solve another minimization problem, is quite an unusual one. But by a few steps this constraint may be written in a standard form which can be handled by optimization software. The first of these steps is the linearization of the objective functions.

Take the second stage minimization problem

$$
\vec{y}^{s}=\operatorname{argmin} \sum_{(i j) \in A} c_{i j}\left(y_{i j}^{s}\right)^{2}
$$

Subject to

$$
\begin{gathered}
y_{i j}^{s} \geq 0 \quad(i j) \in A, s \in S \\
\left(B \vec{y}^{s}\right)_{i}=b_{i}+m_{i}^{s} x_{i}-\left(\vec{m}^{s} \cdot \vec{x}\right) t_{i} \quad i \in N, s \in S .
\end{gathered}
$$

As already observed, these second stage decisions solve \#S independent but very similar convex network flows. (The only difference between scenarios is a smaller or larger change in supply of the wind mills with their corresponding corrections.) The similarity between the scenarios may be used to obtain the solution of all $\# S-1$ network flows efficiently by starting out from the solution to the first one. Of convex network flows it is known that they can be approximated by linear network flows to any desired degree of accuracy by adding multiple arcs between the connected nodes, and defining appropriate arc cost and capacity parameters (as, for example, [19] does). This can be understood as follows.

Suppose some arc $(i j) \in A$ has capacity $u_{i j}$ and cost function $c_{i j}\left(y_{i j}\right)^{2}$, and suppose the cost function is approximated by a piecewise linear function consisting of $H$ line segments as in Fig. 2.

Now this arc $(i j)$ and the piecewise linear cost function on it behave in the same way as $H$ parallel arcs $(i j, h), h=1, \ldots H$ with capacity $U_{i j, h}=u_{i j} / H$ and linear cost functions $C_{i j, h}$ that have a slope equal to the slope of the $h$ 'th line segment in the picture. This follows because a flow from $i$ to $j$ that should minimize the cost, will allways first fill the cheapest arcs ( $i j$, $h$ ), that is, from $h=1$ upwards to $h=H$. The old cost of the flow over arc (ij) now equals the sum of the new flows over the $\operatorname{arcs}(i j, h)$.

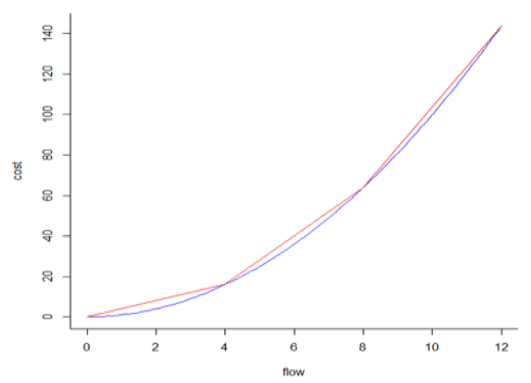

Fig. 2. Linearization of the cost function.

By applying this transformation the number of nodes remains the same, and node balance constraints change only in the sense that there is one more arc parameter to sum over. The objective function becomes

$$
\vec{y}^{s}=\operatorname{argmin} \sum_{(i j \in A)} \sum_{h \in\{1, \ldots, H\}} C_{i j, h} y_{i j, h} .
$$

The value of the first stage objective function $\left(y_{i j}^{s}\right)^{2} r_{i j}$ can easily be computed in terms of the new flow variables $y_{i j, k}$ and in a similar way newly defined $R_{i j, k}$. The total linearized problem then looks like:

$$
\text { Minimize } \quad \sum_{s \in S} p^{s} \times\left(\sum_{(i j) \in A} \sum_{h \in\{1, \ldots, H\}} R_{i j, h} y_{i j, h}^{s}\right)
$$

Subject to

$$
\begin{gathered}
x_{i} \in\{0,1, \ldots\} \quad i \in N \\
\sum_{i \in N} x_{i}=l
\end{gathered}
$$

and where

$$
\vec{y}^{s}=\operatorname{argmin} \sum_{(i j \in A)} \sum_{h \in\{1, \ldots, H\}} C_{i j, h} y_{i j, h}^{s}
$$

Subject to

$$
\begin{gathered}
0 \leq y_{i j, h}^{s} \leq U_{i j, h} \quad(i j, h) \in A^{*}, s \in S \\
\sum_{j, h:(i j, h) \in A^{*}} y_{i j, h}^{s}-\sum_{j, h:(j i, h) \in A^{*}} y_{i j, h}^{s} \\
=b_{i}+m_{i}^{s} x_{i}-\left(\vec{m}^{s} \cdot \vec{x}\right) t_{i} \quad i \in N, s \in S,
\end{gathered}
$$

where $A *$ denotes the new (strongly enlarged) set of arcs with linear cost, indexed by $(i j, h)$, where $(i j) \in A, h \in\{1, \ldots, H\}$. Again the last equation represents the node balance constraints. By the introduction of the parallel arcs, each node now has more arcs incident to it than before, but the structure of the graph remains the same. If $B^{*}$ denotes the node-arc incidence matrix for the new set of arcs, the flow balance constraints could have been written $\left(B^{*} \vec{y}^{s}\right)_{i}=b_{i}+m_{i}^{s} x_{i}-\left(\vec{m}^{s} \times \vec{x}\right) t_{i}$ for $i \in N, s \in S$.

\section{Reformulating the Second Stage Constraint}

The second and final step of rewriting the problem is performed in this section. When this has been done the problem of minimizing the transportation losses will be formulated in the form of a standard mathematical program.

The point of linearizing the network flow is that for linear network flows a well-known duality framework exists, in which strong duality holds: solving the minimum cost flow under flow nonnegativity and balance constraints is equivalent to (and yields the same optimal objective value as) solving another, related, linear program, called its dual. [22] give a very nice introduction into general duality in Section IV, and apply it to network flows in Section VII. Alternatively [19], chapter 9 derive the dual network problem from first principles.

The general results may be summarized by the following. Let $z\left(y^{*}\right)$ denote the cost value of a minimum cost flow with flow variables $\vec{y}$ and balance vector $\overrightarrow{\tilde{b}}$. Define $\pi: N \rightarrow R$, called the node potential which associates with every node $i$ a real number $\pi_{i}$. Then maximizing $w(\pi)=\sum_{i \in N} \tilde{b}_{i} \pi_{i}-\sum_{(i j) \in A} \max \left(0, \pi_{i}-\pi_{j}-c_{i j}\right) u_{i j}$ yields an optimal node potential $\pi^{*}$, and $w\left(\pi^{*}\right)=z\left(y^{*}\right)$.Therefore, when faced with a linear network flow problem, it can be checked if it has been solved to optimality by a simple condition: does a node potential $\pi$ exist for which $w(\pi)$ equals the current flow cost? 
This theory can be applied in the following way. In the two stage model above, one of the constraints is that the $\vec{y}^{s}$ have to be chosen such that they minimize some flow cost objective $\sum_{i j, h \in A^{*}} C_{i j, h} y_{i j, h}^{s}$ called the primal value. For the optimal $\vec{y}^{s}$, there exist node potentials $\vec{\pi}^{s}$ for which the dual value $w\left(\pi^{*}\right)$ equals the primal. Hence instead of the 'argmin'-constraint, it is equivalent to add free node potential variables for every scenario, and add the constraint that for every scenario primal value should equal dual value.

Let $\tilde{b}_{i}^{s}=b_{i}+m_{i}^{s} x_{i}-\left(\vec{m}^{s} \times \vec{x}\right) t_{i}$ be the balance at node $i$, corrected for scenario $s$ and the placement decisions $\vec{x}$. Then the condition that $\vec{y}^{s}=\arg \min \sum_{i j, h \in A^{*}} C_{i j, h} y_{i j, h}$, where $\vec{y}^{s}$ is a feasible flow, is equivalent to the condition that

$$
\begin{aligned}
& \sum_{i \in N} \tilde{b}_{i}^{s} \pi_{i}^{s}-\sum_{(i j, h) \in A^{*}} \max \left(0, \pi_{i}^{s}-\pi_{j}^{s}-C_{i j, h}\right) U_{i j, h} \\
& =\sum_{i j, h \in A^{*}} C_{i j, h} y_{i j, h}
\end{aligned}
$$

where $\vec{y}^{s}$ is a feasible flow and $\vec{\pi}^{s}$ a node potential vector. After these new variables are introduced and the constraint changed as described above, the model can finally be formulated as a standard optimization problem:

$$
\text { Minimize } \quad \sum_{s \in S} p^{s} \times\left(\sum_{i j, h \in A^{*}} R_{i j, h} y_{i j, h}^{s}\right)
$$

Subject to

$$
\begin{gathered}
x_{i} \in\{0,1, \ldots\} \quad i \in N \\
\sum_{i} x_{i}=l \\
0 \leq y_{i j, h}^{s} \leq U_{i j, h} \quad(i j, h) \in A^{*}, s \in S \\
\pi_{i}^{s} \in R \quad i \in N, s \in S \\
\left(B^{*} \vec{y}^{s}\right)_{i}=b_{i}+m_{i}^{s} x_{i}-\left(\vec{m}^{s} \cdot \vec{x}\right) t_{i} \quad i \in N, s \in S \\
\sum_{i \in N} \tilde{b}_{i}^{s} \pi_{i}^{s}-\sum_{(i j, h) \in A^{*}} \max \left(0, \pi_{i}^{s}-\pi_{j}^{s}-C_{i j, h}\right) U_{i j, h} \\
=\sum_{i j, h \in A^{*}} C_{i j, h} y_{i j, h}^{s} \quad s \in S
\end{gathered}
$$

where $\tilde{b}_{i}^{s}$ denotes the corrected node balances, and $B^{*}$ the node-arc incidence matrix adjusted for the parallel arcs.

\section{Solution Strategies}

With respect to the solution of the mathematical program formulated in the last section, there are a few things that should be kept in mind. By the introduction of the new indices $s$ for stochastic programming and $h$ for linearization of the convex cost arcs, and the introduction of the new variables $\pi^{s}$, the whole program quickly becomes giant. For example, in the next section, 729 scenarios are defined, that is, $\# S=729$, and if a transmission line of heat capacity 1000 MVA is to be modelled with precision $1 \mathrm{MW}$, then $H=100$. The result is, that the number of flow variables is multiplied by almost a million, and that in addition \#S.n new (node potential) variables enter the problem.

But there is one more (somewhat hidden) drawback of the method described in this section. The constraint that the dual must equal the primal value of the second stage was written

$$
\begin{gathered}
\sum_{i \in N} \tilde{b}_{i}^{s} \pi_{i}^{s}-\sum_{(i j, h) \in A^{*}} \max \left(0, \pi_{i}^{s}-\pi_{j}^{s}-C_{i j, h}\right) U_{i j, h} \\
=\sum_{i j, h \in A^{*}} C_{i j, h} y_{i j, h}^{s}, s \in S
\end{gathered}
$$

But the balance $\tilde{b}_{i}^{s}$ used in this equation crucially depends on the first stage decisions $\vec{x}$. It was defined $\tilde{b}_{i}^{s}=b_{i}+m_{i}^{s} x_{i}-\left(\vec{m}^{s} \times \vec{x}\right) t_{i}$, and therefore the product $\tilde{b}_{i}^{s} \pi_{i}^{s}$ contains a term $x_{i} \pi_{i}^{s}$; which makes the model nonlinear. Naive rigorous solution when the model is defined on any realistic scale (which may include hundreds of nodes and arcs, as well as hundreds of scenarios and parallel arcs) will probably be infeasible. Specialized algorithms may be designed by exploiting the primal-dual relationship between variables $y_{i j, h}^{s}$ and $\pi_{i}^{s}$, but are beyond the scope of this paper.

Therefore here we present a heuristic approach, based on the observation that the functions $\sum_{i j} r_{i j} p_{i j}^{2}$ and $\sum_{i j} x_{i j} p_{i j}^{2}$ have the same structure. If for all branches $i j$, the fraction $x_{i j} / r_{i j}$ is the same, then minimization of $\sum_{i j} x_{i j} p_{i j}^{2}$ would immediately yield a placement that minimizes losses. Now minimize objective function $\sum_{i j} c_{i j} p_{i j}^{2}$, where the cost parameters $c_{i j}$ are a mixture of reactance $x_{i j}$ and resistance $r_{i j}$. Minimization of $\sum_{i j} x_{i j} p_{i j}^{2}$ is needed for correct power flows; minimization of $\sum_{i j} r_{i j} p_{i j}^{2}$ yields loss minimizing objective. So a certain tradeoff has to be made which aspect is more important for an arc. One simple suggestion could be to take cost parameters $c_{i j}=\left(r_{i j}+x_{i j}\right) / 2$ or another: $c_{i j^{\prime}}=r_{i j^{\prime}} \times\left(\frac{1}{m} \sum_{i j} \frac{r_{i j}}{x_{i j}}\right)^{-1}$

Now the second stage of the problem disappears, which makes the problem much easier. Two extreme parameter choices can be identified. First: disregard resistances completely (that is, take $c_{i j}=x_{i j}$ ), which respects power flows, but disregards losses ${ }^{6}$. And second: disregard reactances completely (that is, take $c_{i j}=x_{i j}$ ), which does minimize losses, but does not respect power flows. Ref. [15] makes this second choice (he does not respect power flows; only flow balances). It is difficult to say something intuitive about the quality performance of this heuristic; in general it will perform poorer if $x_{i j} / r_{i j}$ differs much for different arcs.

\section{WIND POWER SIMULATION}

We performed a simulation of wind speeds in the Netherlands, based on empirical data and on wind speed models from literature. The goal is to compute reasonable values for the model parameters $p^{s}$ and $m^{s}$ that were used in the optimization model. Recall that the interpretation of $p^{s}$ is the probability of a scenario $s \in S$, whereas $m^{s}$ is a vector

\footnotetext{
${ }^{6}$ This is the choice made to produce the results in the next section.
} 
containing for scenario $s$ the joint wind power output if on every node there would be exactly one wind turbine.

For this paper data were taken from the hourly wind speed measurements of all 50 measurement stations during the years 2001 until 2010 from The Dutch meteorological institute (www.knmi.nl).

First we made some partition of the Netherlands into a few different wind zones, within which we will assume equal wind speeds based on clustering techniques, exact description can be found in [23]. Next we modelled the wind speed at a particular site. In literature, the Weibull or even Rayleigh (which is a special case of the Weibull) distribution is advocated. See, for example, [12], or [14]. Conclusion of our analysis is that the assumption of Rayleigh distributed marginals is sufficiently justified. Next we looked at the joint distribution of the wind speeds in the several regions. Ref. [12] suggests a multivariate distribution which is easy to simulate, and has marginals with approximate Rayleigh properties. They cite [24] and [25] as mathematically more precise, but of a (for this purpose) unjustified complexity.

Having simulated 10000 independent instances of joint wind speeds, these should now be transformed into the powers that are produced by a wind turbine within these zones. Four main characteristics are mentioned in literature: a wind speed value $v_{c i}$ (cut-in velocity) below which the turbine has no output, a rated velocity $v_{r}$ above which the turbine has its maximum output, and a cut-out velocity $v_{c o}$ above which the turbine is deactivated in order to prevent damage. The fourth characteristic is the rated or maximum power $P_{r}$ itself.

Ref. [15] takes as power output curve the first graph in Fig. 3 , which is doubtless the more accurate model. But we think that the linear approximation used by [14] and [13] suffices for our purposes.

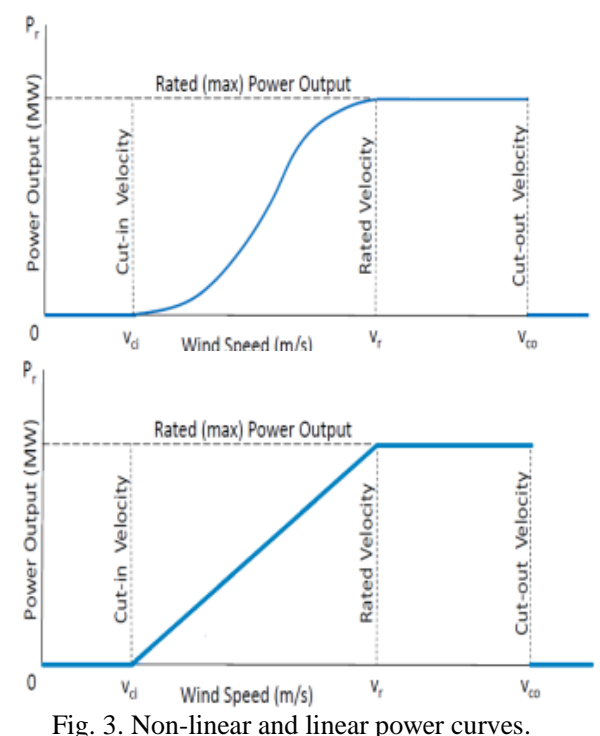

There are many different sizes and types of wind turbines available in industry. For this paper typical characteristics for a modern large $2500 \mathrm{~kW}$ turbine were taken.

By the procedure described until here, it is possible to simulate correctly correlated wind power outputs for single wind plants over the different regions. Total wind power output in a region can then be obtained by multiplying that value by the number of turbines in the region, as described in the previous section. This simulation procedure can be used to compute values for the scenario parameters $p^{s}$ and $m^{s}$, defined for the stochastic optimization program.

$p^{s}$, the probability of a scenario $s \in S$, could be computed by partitioning the 6-dimensional range of power outputs, and counting which parts of the simulated values would lie in the particular areas. $m^{s}$, the value of the scenario, is then obtained by averaging over these values only. But the fact that we started the simulation from independent uniforms, provides us with an opportunity for simplification: by simply partitioning the range of the multivariate uniforms (that is, $\left.[0,1]^{6}\right)$, then mapping them to output powers and compute the $m^{s}$, it becomes easy to define scenarios which have all equal probability $p^{s}$.

Now that these parameters $p^{s}$ and $m^{s}, s \in S$ have been computed, the deterministic optimization problem that was drawn up in the previous section can be solved.

\section{RESULTS}

In this section the results of the performed computations are presented. In order to visualize the trade-offs mentioned in Section I, first some preliminary results are shown: the trade-off between high expected value and small variance of the total wind power, is illustrated in Section VII Part A and the trade-off between local and central placement for stochastic supply, is illustrated in Section VII Part B. In the last subsection the results of the full model are shown.

\section{A. Significance of Wind Zones}

In Fig. 4, normalized output power histograms are shown for 6 wind turbines. The left picture applies to the situation where all outputs are $100 \%$ correlated, i.e., all turbines are in a single zone. The region of Groningen and Friesland is taken as an example, because it has medium expected wind speed. The right picture applies when the turbines are equally distributed over 6 different wind zones. The results are obtained from the data used and discussed in Section VI.
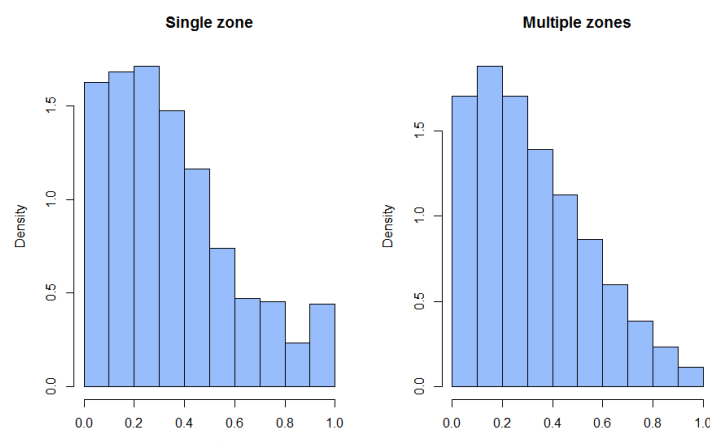

Fig. 4. Histograms for power output in single and multiple zones.

Although the effect is not very strong, one can see that the distribution for the single zone is much flatter than the distribution for multiple zones. In fact, the variance of the single zone output is 1.5 times higher than for multiple zones. This suggests that there could be benefits from spreading wind turbines over the different wind zones. Of course the benefits in larger countries would be greater (for example, [15] presents similar histograms for various sites in the United States, indicating a stronger effect in variance reduction).

Now suppose that we distribute 20 wind turbines over the 
various zones, yielding a placement vector $p$ which contains for every zone $i=1, \ldots, 6$ the number of wind turbines $p_{i}$ that are allocated to that zone. Define the output of a single wind turbine in zone $i$ as $X_{i}, i=1, \ldots, 6$ and define the total power output of the 20 turbines $X_{p}=\sum_{i=1}^{6} p_{i} X_{i}$.

For various reasons a high variance of the output could be undesirable. In our context high variance of power output would mean that it is more difficult to match it to local demand, resulting in higher power losses by transportation. In finance and trade, high variance of production means less predictable volumes to be traded, resulting in lower selling prices. In order to compare expected value and variance, it has been proposed (for example [26]) to maximize the function $f(p)=E\left[X_{p}\right]-\lambda \operatorname{Var}\left(X_{p}\right)$, where $\lambda$ represents the costs of volatility. This so-called mean -variance model is common in financial optimization and other disciplines. The variance of $X_{p}$ can be expressed as

$$
\begin{gathered}
\operatorname{var}\left(X_{p}\right)=\operatorname{var}\left(\sum_{i=1}^{6} p_{i} X_{i}\right) \\
=\sum_{i=1}^{6} \sum_{j=1}^{6} \operatorname{cov}\left(p_{i} X_{i}, p_{j} X_{j}\right)=\sum_{i=1}^{6} \sum_{j=1}^{6} p_{i} p_{j} \operatorname{cov}\left(X_{i}, X_{j}\right),
\end{gathered}
$$

where the covariance matrix $\sum$ of the $X_{i}$ is known from the wind data discussed in Section VI. Let $\mu_{i}=E\left[X_{i}\right]$, and let $\sigma_{i, j}=\operatorname{cov}\left(X_{i}, X_{j}\right)$ be fixed parameters. Then the function $f(p)$ can be written in the nonlinear form

$$
f(p)=\sum_{i=1}^{6} p_{i} \mu_{i}-\lambda \sum_{i=1}^{6} \sum_{j=1}^{6} p_{i} p_{j} \cdot \sigma_{i, j},
$$

and its variables $p_{i}, i=1, \ldots, 6$ are subject to the constraints $p_{i} \in\{0, \ldots, 20\}$, and $\sum_{i=1}^{6} p_{i}=20$. The parameters are given by

$$
\mu=\left(\begin{array}{l}
65.77 \\
56.95 \\
37.00 \\
41.74 \\
41.18 \\
50.47
\end{array}\right)
$$

and

$$
\Sigma=\left(\begin{array}{llllll}
1008.7 & 699.09 & 480.54 & 542.84 & 585.35 & 538.84 \\
699.09 & 781.24 & 415.07 & 438.21 & 455.23 & 569.42 \\
480.54 & 415.07 & 389.71 & 381.42 & 394.28 & 377.39 \\
542.84 & 438.21 & 381.42 & 526.13 & 479.52 & 409.67 \\
585.35 & 455.23 & 394.28 & 479.52 & 591.54 & 412.97 \\
538.84 & 569.41 & 377.39 & 409.67 & 412.97 & 678.27
\end{array}\right)
$$

Again Aimms can solve this for various $\lambda$. The problem is a Mixed Integer Quadratic Programming (MIQP) problem with 6 variables. Table I shows results for various values of $\lambda$. Because of the high values in matrix $\sum$ compared to $\mu$, the range of $\lambda$ for which interesting changes occur, is quite near to zero $(\lambda \in[0,0.01])$.
TABLE I: DISTRIBUTION OF TURBINES FOR VARIOUS $\lambda$ IN THE MEAN VARIANCE MODEL

\begin{tabular}{|l|l|l|l|l|l|l|l|l|l|}
\hline $100 \lambda$ & 0 & 0.1 & 0.2 & 0.3 & 0.4 & 0.5 & 0.6 & 0.7 & 1 \\
\hline North Sea Coast & 20 & 15 & 9 & 4 & 2 & 0 & 0 & 0 & 0 \\
\hline Zuiderzee & 0 & 4 & 4 & 3 & 3 & 2 & 2 & 1 & 0 \\
\hline Inlands & 0 & 0 & 0 & 6 & 9 & 11 & 13 & 14 & 15 \\
\hline Limburg & 0 & 0 & 0 & 2 & 2 & 3 & 2 & 2 & 2 \\
\hline WN Brabant & 0 & 0 & 0 & 0 & 0 & 0 & 0 & 0 & 0 \\
\hline Groningen Friesl & 0 & 1 & 7 & 5 & 4 & 4 & 3 & 3 & 3 \\
\hline
\end{tabular}

For $\lambda=0$, naturally all turbines are placed in the zone with highest average wind speed. For $\lambda$ too high, the term $-\lambda \operatorname{Var}\left(X_{p}\right)$ becomes dominant, and most turbines are allocated to the zone 'Inlands'. This zone has lowest variance, but it has also low expected value.

The value of $\lambda$ that should be taken for practical optimization is determined by external factors (market volatility, share of wind power in the generation portfolio of the power company). One can clearly observe that the optimal wind mill distribution for a power company strongly depends on the value of its $\lambda$.

\section{B. Network Structure}

In the former section only wind speed behaviour was taken into account, while network structure of the power grid was neglected. An optimal dispersion over the various wind zones was achieved, depending on the relative importance of expectation and variance of the output. In this section, by contrast, the structure of the power grid is analysed. This is done by placing Distributed Generation (DG) units in the network, and assessing their effect on the total line losses on the grid. Optimal locations for both deterministic and stochastic DG units are presented. The problem is similar to the placement of wind turbines, but simpler because wind speed correlation structure is disregarded.

As a first orientation into the structure and the load of the HV grid, the simple power flow problem can be solved. It yields the following observations:

1) The global structure of the grid resembles two connected rings, as depicted in the upper part of Fig. 5. The province of Groningen and the loose ends in the west are exceptions to this, but the major part of the transportation is taking place at one cycle (mainly from West to East).

2) For achieving minimum transportation losses, DG units are very likely placed on nodes with negative supply. Therefore if node 619 (Diemen), which has positive supply, is moved to the wind zone 'North Sea Coast', this is likely to have no consequences for any solutions.

3) Almost all nodes in 'North Sea Coast' and in 'West Noord-Brabant' are supply nodes. ${ }^{7}$ It is unattractive to place DG units there. When these two zones are united, and the rest of the nodes are sorted according to their wind zones, a picture can be made of the power exchanges between the several zones. This is shown in the lower part of Fig. 5. Net supplies are cumputed per zone, and the power exchange between the various zones is the sum over one or two transmission lines.

\footnotetext{
${ }^{7}$ The large supply at Beverwijk is explained by offshore wind farms at the height of Noord Holland. The large supplies at Maasvlakte and Geertruidenberg are explained by the large number of Combined Heat and Power (CHP) systems used by greenhouses and industry. Borssele is home to a nuclear power plant.
} 
Both quantities are in MW.

4) From these pictures it is intuitively clear that DG units will be placed most likely in the 'Inlands' or 'Limburg'.

5) Total load on the grid is $13325 \mathrm{MW}$. About half of it is exchanged between the zones. The network losses for the standard power flow solution amount to 7.5 MW.

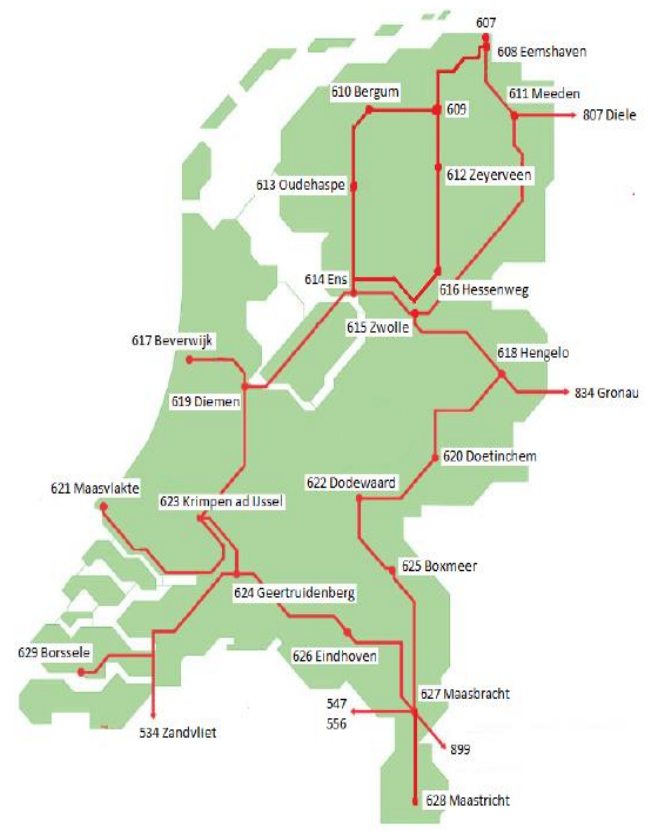

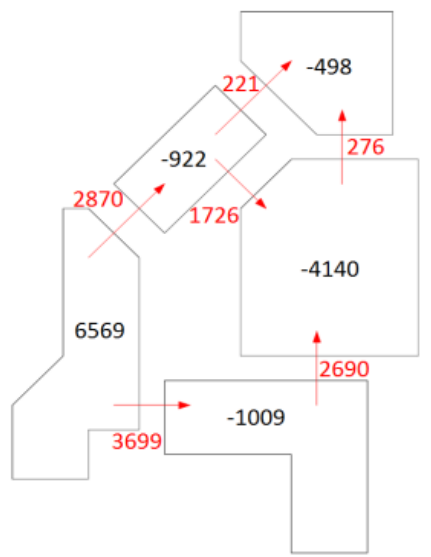

Fig. 5. Power exchanges in MW between the wind zones for the standard power flow solution.

In order to investigate network preferences for the placement of DG, Tables II and III are presented. They contain the results of a sequence of placement problems for varying DG penetration, and for varying size of the generation units. By penetration is meant: total added generation capacity divided by total original generation capacity (13325 MW).

In the limit of $100 \%$ penetration and small enough unit size, it is clear that transportation losses can be made to vanish. When distributed generation equals demand at every node, no power transportation takes place at all.

TABLE II: DISPERSION OF DETERMINISTIC DG OVER WIND ZONES

\begin{tabular}{|lcccccccc|}
\hline \#DG units & 30 & 60 & 120 & 6 & 12 & 24 & 3 & 6 \\
\hline $\begin{array}{l}\text { Unit size } \\
(\mathrm{MW})\end{array}$ & 100 & 100 & 100 & 500 & 500 & 500 & 2000 & 2000 \\
\hline $\begin{array}{l}\text { total DG } \\
\text { capacity(MW) }\end{array}$ & 3000 & 6000 & 12000 & 3000 & 6000 & 12000 & 6000 & 12000 \\
\hline \hline $\begin{array}{l}\text { Coast and } \\
\text { Brabant }\end{array}$ & 0 & 0 & 1 & 0 & 0 & 0 & 0 & 0 \\
\hline Zuiderzee & 0 & 0 & 6 & 0 & 0 & 1 & 0 & 0 \\
\hline Inlands & 29 & 41 & 44 & 5 & 8 & 9 & 3 & 2 \\
\hline Limburg & 0 & 9 & 48 & 0 & 2 & 10 & 0 & 3 \\
\hline $\begin{array}{l}\text { Groningen } \\
\text { Friesland }\end{array}$ & 1 & 10 & 18 & 1 & 2 & 4 & 0 & 1 \\
\hline \hline $\begin{array}{l}\text { Network } \\
\text { losses(MW) }\end{array}$ & 3.71 & 1.60 & 0.04 & 3.72 & 1.59 & 0.06 & 1.82 & 0.46 \\
\hline
\end{tabular}

TABLE III: DISPERSION OF STOCHASTIC DG OVER WIND ZONES

\begin{tabular}{|lllllllll|}
\hline \#DG units & 30 & 60 & 120 & 6 & 12 & 24 & 3 & 6 \\
\hline $\begin{array}{l}\text { mean unit } \\
\text { output (MW) }\end{array}$ & 100 & 100 & 100 & 500 & 500 & 500 & 2000 & 2000 \\
\hline $\begin{array}{l}\text { mean DG } \\
\text { capacity(MW) }\end{array}$ & 3000 & 6000 & 12000 & 3000 & 6000 & 12000 & 6000 & 12000 \\
\hline \hline $\begin{array}{l}\text { Coast and } \\
\text { Brabant }\end{array}$ & 0 & 0 & 22 & 0 & 0 & 5 & 0 & 1 \\
\hline Zuiderzee & 0 & 1 & 6 & 0 & 0 & 1 & 0 & 0 \\
\hline Inlands & 24 & 27 & 29 & 5 & 5 & 6 & 2 & 1 \\
\hline Limburg & 2 & 22 & 47 & 0 & 5 & 9 & 1 & 3 \\
\hline $\begin{array}{l}\text { Groningen } \\
\text { Friesland }\end{array}$ & 4 & 10 & 16 & 1 & 2 & 3 & 0 & 1 \\
\hline \hline $\begin{array}{l}\text { Network } \\
\text { losses(MW) }\end{array}$ & 4.287 & 3.17 & 3.0 & 4.3 & 3.209 & 3.0 & 3.651 & 4.055 \\
\hline
\end{tabular}

The extreme case of placing only one DG unit can be used to obtain the notion of a kind of centrality of a node. When placing one unit of $1 \mathrm{MW}$, the location which minimizes the loss is the point for which the network losses are most sensitive to a change in demand. One DG unit with $100 \%$ penetration yields the best location if the whole network would be powered from one source node. ${ }^{8}$ The resulting

${ }^{8}$ Note that this is purely theoretical because of the following. In our model extra DG power is compensated by decreasing supply of the original supply nodes. This choice was made in order to approximate the situation in which all commercial power plants in the country take equal share in the compensation. When the share of DG becomes too large, this approximation fails. 
source node turns out to be Doetinchem for small size, Dodewaard when a size of $6000 \mathrm{MW}$ is reached, and Boxmeer when $100 \%$ penetration is approached.

The computations were done using the heuristic described in 5.4. The results in Table II confirm the intuition that both smaller unit size and increasing DG penetration have positive effect on transportation losses.

The most remarkable feature of these results is the enormous sensitivity of the total losses to DG. Even for small penetration the losses drop to half the original value. Certainly for lower penetration, the size of the DG units makes little difference. Next it is notable that the first DG units are mostly placed in the inlands, but at a certain point Limburg takes over the central role.

The same computations were done for stochastic DG units: a placement is found for which expected transportation losses are minimized. For each case, the expected output of the DG units equals the size of its deterministic equivalent in the table above. Three scenarios were used: one with zero DG output, one with its expected output, and one with output of twice the expectation. The results are presented in Table III.

It immediately stands out that the strong positive effect of deterministic DG on the transportation losses, is partly neutralized when DG units are stochastic. Interestingly, all penetrations yield comparable resulting net losses. Furthermore, stochastic DG units seem to be more dispersed than their deterministic equivalents. The strongest dispersion effect is seen for high penetration. However, it should be observed that for high expected penetration, there is excess of DG power in the third scenario (where all units produce twice their mean output). This results in the situation that the original supply nodes become demand nodes, making the North Sea Coast an attractive zone to place the DG units. The results for this situation should be distrusted.

\section{Wind Turbine Placement}

In this section results of the solutions to the wind turbine placement problem are presented and discussed. The computations were done using the heuristic discussed in 5.4. Recall that the maximum output power of the turbine that was regarded equals 2.5 MW. The network results presented in the former section make clear that this quantity is almost negligible compared to the total load. Therefore, the output power was multiplied by successively 40, 200 and 800 in order to obtain interesting results. That is, the 'DG units' of maximum unit output of $100 \mathrm{MW}, 500 \mathrm{MW}$ and $2000 \mathrm{MW}$ correspond to groups of 40, 200 and 800 large wind mills.

The 729 scenarios that were simulated in Section VI were used. These wind power scenarios reflect mutual correlation between the zones, and the Rayleigh distributed wind speeds for each zone apart. The resulting mathematical program is a mixed integer quadratic program (MIQP) with 64155 constraints and 88240 variables, of which 29 integer. Aimms' cplex 12.4 solver is able to solve this within a few minutes on a standard PC. It can be observed that both unit size and number of units have a negative influence on the computation time. (A larger number of units increase the number of basic feasible solutions strongly. It is less evident why larger unit size requires longer computation time.)

Consider the wind turbine placement problem. Placement of 30 wind turbines with maximum output of $100 \mathrm{MW}$ yields the result that all turbines are placed in Groningen, the average total output being less than $600 \mathrm{MW}$. This shows that the solution of the wind turbine placement problem may differ from what one might expect when just wind or network data are considered. Highest wind speeds are to be found at the North Sea Coast, but there are no demand nodes there. Second highest wind speeds occur in the 'Zuiderzee' region which does have a shortage of $922 \mathrm{MW}$. But apparently lower production in Groningen can cause a greater decrease in power transportation than a somewhat higher production in the 'Zuiderzee' region would cause. However for the Inlands and Limburg (which have an even more attractive position on the network for DG placement) wind speed is just too low. Table IV shows some results for higher penetrations.

TABLE IV: DiSPERSION OF WIND TURBINES OVER WIND ZONES. IN THE LOWEST ROW SOME COMPUTATION TIMES ARE DiSPLAYED, WHICH IT TOOK AIMMS 3.12 ON A COMPUTER WITH 4GB RAM, INTEl DuO CORE 3.0 GHZ PROCESSOR, RuNNING UNDER 64-BIT WindOWS 7.

\begin{tabular}{|lcccccccc|}
\hline \#wind turbines & 60 & 120 & 240 & 12 & 24 & 48 & 3 & 6 \\
\hline max unit output(MW) & 100 & 100 & 100 & 500 & 500 & 500 & 2000 & 2000 \\
\hline mean DG capacity(MW) & 1120 & 2060 & 3710 & 1140 & 2072 & 3750 & 1140 & 2140 \\
\hline \hline Coast and Brabant & 0 & 0 & 0 & 0 & 0 & 0 & 0 & 0 \\
\hline Zuiderzee & 0 & 0 & 7 & 0 & 0 & 2 & 0 & 0 \\
\hline Inlands & 0 & 1 & 57 & 0 & 0 & 11 & 0 & 0 \\
\hline Limburg & 23 & 70 & 120 & 4 & 14 & 24 & 1 & 3 \\
\hline Groningen Friesland & 37 & 49 & 56 & 8 & 10 & 11 & 2 & 3 \\
\hline \hline Network losses(MW) & 6.309 & 5.438 & 4.282 & 6.829 & 5.43 & 4.272 & 6.829 & 5.448 \\
\hline Solution time & $157 \mathrm{~s}$ & - & $341 \mathrm{~s}$ & - & - & - & $374 \mathrm{~s}$ & $524 \mathrm{~s}$ \\
\hline
\end{tabular}

It is interesting to see how the Inlands, which seemed so attractive both in the mean variance model for wind power, and in the network structure analysis, are less attractive in the wind turbine placement problem. Units are placed in the Inlands only after Groningen has reached a kind of saturation, and there are already many wind turbines in Limburg. This phenomenon might occur because the expected wind generation is simply too low in the Inlands; but seeing that the difference in expected outputs between Inlands and Limburg is smaller than between Groningen and Zuiderzee, some other reason seems likely.

When there are more than 30 units of $100 \mathrm{MW}$ (that is,
$3000 \mathrm{MW}$ of installed wind capacity) in Groningen, there is excess of wind power in that region during the strong wind scenarios. Some of that power will flow to the neighbouring Inlands. Consequently, Limburg is more attractive than the Inlands because of its greater distance to Groningen. Also neighbouring zones do not have full correlations: highest wind generation scenarios of one zone do not necessarily coincide with highest generation in the other zone. The probability of local power excess may therefore be decreased by placing units in the neighbouring zone. This may explain why the results for wind turbine placement show a higher spreading of generation units than the stochastic DG 
placement regarded in the former section (where correlations were $100 \%$ ).

\section{SUMMARY AND CONCLUSIONS}

In this section the content of this paper is summarized and conclusions are drawn from the computational results. In this paper an introduction was provided into power grid structures and into modelling of power flows with network losses. A mathematical model was formulated in order to capture the wind power Generation Expansion Planning problem for loss minimization in a so-called two stage stochastic program. Network flow duality was applied such as to arrive at a NLP formulation for the stochastic Generation Expansion Planning problem for loss minimization. Rigorous solution algorithms for this NLP problem were outside the scope of this paper. Therefore a simple heuristic was presented, together with a procedure to simulate appropriate scenarios for steady state wind power studies in multiple areas. This implementation of the model was used to generate results for the Dutch HV network.

From the computational results it was possible to conclude that:

1) The effect of reducing transmission losses by placing DG units is significantly weaker for stochastic than for deterministic units;

2) In order to achieve high transmission efficiency, stochastic DG units should be placed further apart than deterministic DG units;

3) There exists a non-trivial relation between the locations of wind turbines and transportation losses, but that interesting effects occur only for higher penetration;

4) The optimal distribution of wind energy over the Netherlands shows a higher spreading than can be explained merely from its stochasticity;

5) The location dependent nature of wind energy is relevant for its effect on power grid performance;

6) Because of the load situation of the Dutch HV grid Groningen and Friesland are the only regions with both attractive wind speeds, and where placement of wind turbines would result in reduction of transmission losses in the HV grid;

7) At the current scale of wind energy, a spreading strategy for wind turbines does not seem to be relevant, neither from a commercial point of view (variance reduction of total output for the Dutch market) nor from a technical point of view (reducing transmission losses) ${ }^{9}$;

8) If in a future scenario wind energy were to acquire a significant share in HV power flows, location selection may become important for grid performance.

Apart from the computational results presented in this paper, some general observations can be made:

1) The loss minimizing uses of DG seem more relevant in large countries like US or Australia, than in a smaller and densely populated country like the Netherlands.

2) Location selection of new wind turbines is done by individual commercial power companies, whereas costs

\footnotetext{
${ }^{9}$ This concerns the HV grid: whether spreading strategies on a MV network scale would be desirable from a technical point of view, should be investigated in a different case study.
}

for transportation are paid by all parties equally. Therefore in practice there is no financial incentive which stimulates locations that improve grid efficiency.

3) LMP methods may open the possibility to let the causer of transportation losses pay for the loss. In such a scenario using DG for reducing losses could also have economic value.

\section{REFERENCES}

[1] C. Ababei and R. Kavasseri, "Efficient network reconfiguration using minimum cost maximum flow-based branch exchanges and random walks-based loss estimations," IEEE Transactions on Power Systems, vol. 326, no. 1, 2011.

[2] M. E. Baran and F. F. Wu, "Network reconfiguration in distribution systems for loss reduction and load balancing," IEEE Transactions on Power Delivery, vol. 4, no. 2, 1989.

[3] R. S. Rao and S. V. L. Narasimham, "Optimal capacitor placement in a radial distribution system using plant growth simulation algorithm," World Academy of Science, Engineering and Technology, vol. 45, 2008.

[4] C. Wang and M. H. Nehrir, "Analytical approaches for optimal placement of distributed generation sources in power systems," IEEE Transactions on Power Systems, vol. 19, no. 4, 2004.

[5] A. D. T. Le, M. A. Kashem, M. Negnevitsky, and G. Ledwich, "Optimal Distributed Generation parameters for reducing losses with economic consideration," Research paper for the Australian Research Council, 2007.

[6] V. H. M. Quezada, J. R. Abbad, and T. G. S. Roman, "Assessment of energy distribution losses for increasing penetration of Distributed Generation," IEEE Transactions on Power Systems, vol. 21, no. 2 , 2006.

[7] N. N. Croes, "Impact of distributed energy generation on energy loss: Finding the optimal mix," MSc thesis, University of Groningen and TNO, 2011 .

[8] N. Croes, F. Phillipson, and M. Schreuder, "Tactical congestion management: The optimal mix of decentralised generators in a district,' Integration of Renewables into the Distribution Grid, 2012.

[9] G. A. Orfanos, P. S. Georgilakis, and N. D. Hatziargyriou, "Transmission Expansion Planning of Systems With Increasing Wind Power Integration," 2012.

[10] Z. Hu, X. Zhou, and W. T. Jewel, "Optimal generation expansion planning with integration of variable renewables and bulk energy storage systems," in Proc. 2013 1st IEEE Conference on Technologies for Sustainability (SusTech), 2013, pp. 1-8.

[11] E Y. Ivanova, N. I. Voropai, and E. Handschin, "A multi-criteria approach to expansion planning of wind power plants in electric power systems," Power Tech, pp. 1-4, 2005.

[12] A. E. Feijoo, J. Cidras, and J. L. G. Dornelas, "Wind speed simulation in wind farms for steady-state security assessment of electrical power systems," IEEE Transactions on Energy Conversions, vol. 14, no. 4, pp. 1582-1588, 1999.

[13] G. Papaefthymiou and B. Kloeck1, "MCMC for Wind Power Simulation," IEEE Transactions on Energy Conversion, vol. 23, no. 1, 2008.

[14] G. Carpinelli, G. Celli, F. Pilo, and A. Russo, "Distributed generation sizing and siting under uncertainty," presented at IEEE Porto Power Tech Conference, 2001.

[15] R. L. Y. Chen, "Models and Algorithms for Stochastic Network Design and Flow Problems: Applications in Truckload Procurement Auctions and Renewable Energy," PhD Thesis, University of Michigan, 2010.

[16] C. Kraemer, K. Goldermann, C. Breue, P. Awate, and A. Moser, "Optimal positioning of renewable energy units," Energy Tech., pp. $1-5,2013$.

[17] K. Purchala, L. Meeus, D. Van Dommelen, and R. Belmans, "Usefulness of DC Power Flow for Active Power Flow Analysis," in Proc. IEEE PES General Meeting, 2005.

[18] D. Crevier, "Approximate Transmission Network Models for Use in Analysis and Design,” MIT Energy Laboratory Report, 1972.

[19] R. K. Ahuja, T. L. Magnanti, and J. B. Orlin, Network Flows, Englewood Cliffs: Prentice-Hall, 1993.

[20] P. Tseng and D. Bertsekas, "An -relaxation method for separable convex cost generalized network flow problems," in Proc. the 5th International IPCO Conference, Vancouver, 1996.

[21] B. Stott, J. Jardim, and O. Alsac, "DC Power Flow Revisited," IEEE Transactions on Power Systems, vol. 24, no. 3, 2009.

[22] D. Bertsimas and J. N. Tsitsiklis, Introduction to Linear Optimization, Athena Scientific, Belmont, Massachusetts, 1997. 
[23] R. A. Johnson and D. W. Wichern, Applied Multivariate Statistical Analysis, Prentice-Hall, Upper Saddle River, 2007.

[24] D. R. Jensen, "Multivariate distributions having Weibull properties," Journal of Multivariate Analysis, vol. 9, pp. 267-277, 1979.

[25] D. R. Jensen, "A generalization of the multivariate Rayleigh distribution," The Indian Journal of Statistics, vol. 32, pp. 193-208, 1970.

[26] M. Steinbach, "Markowitz revisited: Mean-variance models in financial portfolio analysis," SIAM Review, vol. 43, pp. 31-85, 2001.

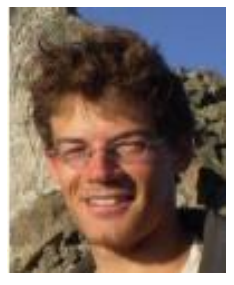

Timo Leenman was born in 1986. He finished his bachelor degree in mathematics at Leiden University, and his master degree in operations research at the University of Amsterdam. The thesis for this master was written at TNO in Delft. His research interests are applying stochastic and non-linear optimization methods to different fields in the Energy sector. Timo is currently working for ORTEC, where his interest has been drawn to planning problems in the Refinery

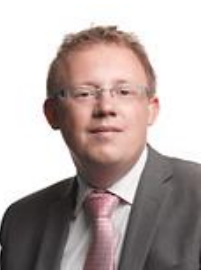

Frank Phillipson was born in 1973. He studied econometrics at the Vrije Universiteit Amsterdam, and wrote his master's thesis in the field of operations research in 1996. In the same year he joined the Delft University of Technology to follow the post-doctoral program 'mathematical design engineering' with a strong focus on application of operations research techniques in networks. From 1998 until 2003 he was employed at KPN Research. In 2002, KPN placed its research department in TNO, the largest applied research institute in the Netherlands, where Frank is currently working in the Department Performance of Networks and Systems. There he is particularly working in the field of planning of ICT/telecom and electricity networks. In addition to this main topic, he has worked on projects for financial and economic models relating to telecom business.

Business. 